\title{
Maximal percolation time in hypercubes under 2-bootstrap percolation
}

\author{
Michał Przykucki \\ Department of Pure Mathematics and Mathematical Statistics \\ University of Cambridge \\ Cambridge CB3 0WB, England, UK \\ m.j.przykucki@dpmms.cam.ac.uk
}

Submitted: Sep 2, 2011; Accepted: May 28, 2012; Published: Jun 13, 2012

Mathematics Subject Classifications: 05D99

\begin{abstract}
Bootstrap percolation is one of the simplest cellular automata. In $r$-bootstrap percolation on a graph $G$, an infection spreads according to the following deterministic rule: infected vertices of $G$ remain infected forever and in consecutive rounds healthy vertices with at least $r$ already infected neighbours become infected. Percolation occurs if eventually every vertex is infected. In this paper we prove that in the case of 2-bootstrap percolation on the $n$-dimensional hypercube the maximal time the process can take to eventually infect the entire vertex set is $\left\lfloor\frac{n^{2}}{3}\right\rfloor$.
\end{abstract}

\section{Introduction}

In this paper we consider the following process known as $r$-bootstrap percolation. Initially a subset $A$ of the set of vertices (called sites when we consider bootstrap percolation) of a graph $G=(V, E)$ is infected and the remaining vertices are healthy. We set $A_{0}=A$ and for $t=1,2,3, \ldots$, we let

$$
A_{t}=A_{t-1} \cup\left\{v \in V(G):\left|N(v) \cap A_{t-1}\right| \geqslant r\right\} .
$$

With $\langle A\rangle=\bigcup_{t=0}^{\infty} A_{t}$ we denote the set of all eventually infected vertices. We say that $A$ percolates if $\langle A\rangle=V(G)$.

Bootstrap percolation, suggested in 1979 by Chalupa, Leith and Reich [8], is a particular example of cellular automata, a concept introduced by von Neumann [11] after a suggestion of Ulam [13]. One way of considering bootstrap percolation is to assume that a set of initially infected sites is chosen in a random way, e.g., all sites are initially infected (i.e., belong to $A$ ) independently with some probability $p$. It is clear that the probability 
of percolation is strictly increasing in $p$ and therefore one of the interesting problems is to determine the critical probability, $p_{c}(G, r)$, defined as follows:

$$
p_{c}(G, r)=\inf \left\{p: \mathbb{P}_{p}(A \text { percolates in } r \text {-bootstrap process on } \mathrm{G}) \geqslant 1 / 2\right\} \text {. }
$$

This problem has been considered by various researchers for many choices of $G$ and $r$, e.g., by Aizenman and Lebowitz [1], Holroyd [9] and by Balogh, Bollobás and Morris [3, 4], who in [5] significantly improved the bounds on the critical probability for 2-bootstrap percolation on $Q_{n}$, the $n$-dimensional hypercube, given earlier by Balogh and Bollobás $[2]$.

Another type of problems that have been considered are extremal properties of the process itself. The size of the smallest percolating sets in $[n]^{d}$ was studied by Pete and a summary of his results can be found in [6]. Recently the question about the maximal size of a minimal percolating set, posed by Bollobás, was studied by Morris [10] for $G=[n]^{2}$ and $r=2$. A similar problem for 2-neighbour bootstrap percolation on a hypercube was answered by Riedl [12]. In this paper we answer another question, also posed by Bollobás, about extremal properties of the infection process when a set of initially infected sites is chosen in a deterministic way. Namely, we prove the following theorem.

Theorem 1. If $A \subset Q_{n}$ percolates, i.e., if $\langle A\rangle=Q_{n}$, then it percolates in at most $\left\lfloor\frac{n^{2}}{3}\right\rfloor$ steps. Moreover, this bound is tight for all $n \in \mathbb{N}$

In other words, if in the definition of bootstrap percolation we set $r=2, A_{0}=A$ and $G=Q_{n}$, then $A_{t}=Q_{n}$ for some $t \leqslant\left\lfloor\frac{n^{2}}{3}\right\rfloor$ whenever $\bigcup_{t=0}^{\infty} A_{t}=Q_{n}$. For $n \in\{1,2,3,4\}$ sets obtaining the maximal percolation time can by found by exhaustive search. For $n \geqslant 5$ a family of optimal sets can be described as follows: a set $A$ infecting $Q_{n}$ in the maximal possible time consists of a small set initializing the process by infecting $Q_{2}$ if $n=2(\bmod$ 3), $Q_{3}$ if $n=0(\bmod 3)$ or $Q_{4}$ if $n=1(\bmod 3)$ in the maximal time, and of pairs of sites each of which prolongs the process by infecting three "additional" dimensions in the maximal possible time. Details of this construction can be found in the proof of Theorem 14. A similar result for $G=[n]^{2}$ and $r=2$ was recently obtained by Benevides and Przykucki [7].

\section{Notation and basic observations}

Let $\mathbb{N}=\{1,2,3, \ldots\}$ denote the set of natural numbers and let $\mathbb{N}_{0}=\mathbb{N} \cup\{0\}$. The $n$-dimensional hypercube $Q_{n}$ is the graph with vertex set $\{0,1\}^{n}$ and edge set $\{\{x, y\}$ : $\left.x, y \in\{0,1\}^{n},\left|\left\{i: x_{i} \neq y_{i}\right\}\right|=1\right\}$. We shall write $Q_{l}$ for any of the $\left(\begin{array}{l}n \\ l\end{array}\right) 2^{n-l}$ subcubes of dimension $l$ in $Q_{n}$. For $x=\left(x_{i}\right)_{1}^{n} \in\{0,1, *\}^{n}$, let $Q^{x}$ be the subcube $\left\{z=\left(z_{i}\right)_{1}^{n} \in\right.$ $\{0,1\}^{n}: z_{i}=x_{i}$ if $\left.x_{i} \neq *\right\}$. Clearly, $x \rightarrow Q^{x}$ gives a $1-1$ correspondence between $\{0,1, *\}^{n}$ and the subcubes of $Q_{n}$. Let $d(0,1)=1, d(0,0)=d(1,1)=d(*, *)=d(0, *)=$ $d(1, *)=0$ be the distance of two coordinates. The distance of two subcubes $Q^{x}, Q^{y}$ in $Q_{n}$ is $d\left(Q^{x}, Q^{y}\right)=\sum_{i=1}^{n} d\left(x_{i}, y_{i}\right)$, where vectors $x, y$ represent subcubes $Q^{x}$ and $Q^{y}$. In the hypercube graph the distance between sets $Q^{x}, Q^{y}$ is also $d\left(Q^{x}, Q^{y}\right)$. 
For $p, q \in \mathbb{N}$ and $y_{0}, y_{1}, \ldots, y_{p} \in\{0,1, *\}$ we denote by $\left[y_{0}\right]^{q}$ the sequence $\left(y_{0}, y_{0}, \ldots, y_{0}\right)$ of length $q$, and by $\overline{\left(y_{1} y_{2} \ldots y_{p}\right)}$ the set of all permutations of the multiset $\left\{y_{1}, \ldots, y_{p}\right\}$. We use a self explanatory notation to concatenate and nest such sequences, e.g.,

$$
[0]^{2} \overline{\left([*]^{2}[1]^{2}\right)} 0=\{00 * * 110,00 * 1 * 10,00 * 11 * 0,001 * * 10,001 * 1 * 0,0011 * * 0\} .
$$

From now on let us consider 2-bootstrap percolation only. A set $A$ is said to be closed under percolation if $\langle A\rangle=A$. Let us recall some simple results from Balogh and Bollobás $[2]$.

Lemma 2. The only subsets of a hypercube that are closed under percolation are those which are a union of disjoint subcubes that are at distance at least 3 from each other.

For vectors $x, y \in\{0,1, *\}^{n}$ set $x \vee y=z=\left(z_{i}\right)$ where $z_{i}=x_{i}$ if $x_{i}=y_{i}$ and $*$ otherwise. It follows from the definition of $\vee$ that $Q^{x}, Q^{y} \subset Q^{z}$.

Lemma 3. For vectors $x, y \in\{0,1, *\}^{n}$ with $d(x, y) \leqslant 2$ we have $\left\langle Q^{x} \cup Q^{y}\right\rangle=Q^{x \vee y}$.

Given an infection process on $Q_{n}$ with an initial set $A \subset Q^{n}$, a subcube $Q_{l} \subset Q_{n}$ is said to be internally spanned if the restriction of the process to $Q_{l}$ fully infects $Q_{l}$, i.e., if $\left\langle A \cap Q_{l}\right\rangle=Q_{l}$.

Lemma 4. Let $A \subset Q_{n}$ be such that $\langle A\rangle=Q_{n}$. Then there is a nested sequence $Q_{0}=$ $Q_{i_{1}}^{x_{i_{1}}} \subset Q_{i_{2}}^{x_{i_{2}}} \subset \ldots \subset Q_{i_{t}}^{x_{i_{t}}}=Q_{n}$, of internally spanned subcubes (with respect to $A$ ), where $2 i_{j}+2 \geqslant i_{j+1}$ for all $j, 0 \leqslant j \leqslant t-1$. Furthermore, for $j \geqslant 2$ each subcube $Q_{i_{j}}^{x_{i_{j}}}$ is spanned by two internally spanned cubes, namely by $Q_{i_{j-1}}^{x_{i_{j-1}}}$ and a subcube $Q_{m_{j-1}}$ of dimension $m_{j-1} \leqslant i_{j-1}$ which is not a member of the sequence.

We call a longest nested sequence of internally spanned cubes as in Lemma 4 a building sequence of the hypercube. For a vector $x \in\{0,1, *\}^{n}$ we define the dimension of $x$ as $\operatorname{dim}(x)=\left|\left\{i: x_{i}=*\right\}\right|$. Obviously, $\operatorname{dim}(x)$ equals $\operatorname{dim}\left(Q^{x}\right)$, the dimension of the cube $Q^{x}$.

For a set of initially infected sites $A$ of a graph $G$ we set $A_{0}=A$ and let $T_{G}(A)=$ $\min \left\{t: A_{t}=\langle A\rangle\right\}$, where the sets $A_{t}$ are defined as in (1). We call $T_{G}(A)$ the spreading time of $A$ in $G$. For the $n$-dimensional hypercube $Q_{n}$ its maximal percolation time is

$$
M(n)=\max _{A:\langle A\rangle=Q_{n}} T_{Q_{n}}(A)
$$

\section{Maximal percolation time}

In this section we shall prove that $M(n)=\left\lfloor\frac{n^{2}}{3}\right\rfloor$ for all $n \in \mathbb{N}$. We start with the following simple lemma.

Lemma 5. For any $n \in \mathbb{N}, M(n) \leqslant M(n+1)$. 
Proof. Let $A$ be such that $\langle A\rangle=Q_{n}$ and $T_{Q_{n}}(A)=M(n)$. Let

$$
\tilde{A}=\left\{\left(a_{1}, \ldots, a_{n}, j\right):\left(a_{1}, \ldots, a_{n}\right) \in A \text { and } j \in\{0,1\}\right\} .
$$

Then $\langle\tilde{A}\rangle=Q_{n+1}$ and $M(n)=T_{Q_{n}}(A)=T_{Q_{n+1}}(\tilde{A}) \leqslant M(n+1)$.

We shall now define a specific norm which at first sight might not be intuitive and seem odd. However, due to the symmetries of the hypercube we shall highly benefit from it and not lose any generality by considering this particular norm. In the following series of lemmas, which will help us understand how infection spreads on $Q_{n}$ depending on the configuration of the set of initially infected sites, the Reader should think of the particular norm of $x$ as of a quantity that reflects (but not always equals to) the sum of distances between $x$ and $S$ and between $x$ and $T$. These six lemmas shall be summarized in Corollary 12.

Let $n \in \mathbb{N}$ and $s, n_{1}, \ldots, n_{s}, d \in \mathbb{N}_{0}$ with $n \geqslant n_{1}+\ldots+n_{s}+d$. For each $x \in\{0,1\}^{n}$ set

$$
\|x\|_{n_{1}, \ldots, n_{s}}^{a_{1} \ldots a_{d}}=\left(\sum_{i=1}^{n-d} x_{i}\right)\left(\prod_{\substack{i=1 \\ n_{i}>0}}^{s} \mathbb{1}_{\left\{\sum_{j=n_{1}+\ldots+n_{i-1}+1}^{n_{1}+\ldots+n_{i}} x_{j}>0\right\}} \prod_{i=1}^{d} \mathbb{1}_{\left\{x_{n-d+i}=a_{i}\right\}}\right) .
$$

Note that, setting $s=0$,

$$
\|x\|^{a_{1} \ldots a_{d}}=\left(\sum_{i=1}^{n-d} x_{i}\right)\left(\prod_{i=1}^{d} \mathbb{1}_{\left\{x_{n-d+i}=a_{i}\right\}}\right),
$$

so that, setting $s=d=0,\|x\|=\sum_{j=1}^{n} x_{j}$. Note crucially that $\|x\|_{n_{1}, \ldots, n_{s}}^{a_{1} \ldots a_{d}}>0$ only if $x$ has at least one 1 in each of the sequences $\left(x_{n_{1}+\ldots+n_{i-1}+1}, \ldots, x_{n_{1}+\ldots+n_{i}}\right)$ for each $i=1, \ldots, s$ with $n_{i}>0$.

Lemma 6. Let $k, l \in \mathbb{N}_{0}, n=k+l$, and set $S=[*]^{k}[0]^{l}$ and $T=[0]^{k}[*]^{l}$ and $A_{0}=S \cup T$. Then

$$
A_{t} \supset\left\{x \in\{0,1\}^{n}:\|x\| \leqslant t+1\right\}=\overline{\left([*]^{t+1}[0]^{k+l-t-1}\right)}
$$

for every $t \in \mathbb{N}$.

Proof. By induction on $t$, noting that $\|x\| \leqslant 1$ implies $x \in A_{0}$, and that every $x \in\{0,1\}^{n}$ with $\|x\|=t+1 \geqslant 2$ has at least two neighbours $y$ with $\|y\|=t$.

Lemma 7. Let $k, l \in \mathbb{N}_{0}, n=k+l+1$, and set $S=[*]^{k}[0]^{l+1}$ and $T=[0]^{k}[*]^{l} 1$ and $A_{0}=S \cup T$. Then

$$
\begin{aligned}
& A_{t} \supset\left([0]^{k+l} *\right) \cup\left\{x \in\{0,1\}^{n}: 1 \leqslant\|x\|^{0} \leqslant t\right\} \cup\left\{x \in\{0,1\}^{n}: 1 \leqslant\|x\|^{1} \leqslant t\right\}= \\
& \overline{\left([*]^{t}[0]^{k+l-t}\right)} 0 \cup \overline{\left([*]^{t}[0]^{k+l-t}\right)} 1
\end{aligned}
$$

for every $t \in \mathbb{N}$. 
Proof. Clearly we have $[0]^{k+l} * \subset S \cup T=A_{0}$. We shall show that both $\|x\|^{0}=1$ and $\|x\|^{1}=1$ imply $x \in A_{1}$. If $\|x\|^{1}=1$ then either $x \in T \subset A_{0}$ or $x_{j}=1$ for some $1 \leqslant j \leqslant k$. In the latter case $x$ has two neighbours in $A_{0}$ : one is $[0]^{k+l} 1$ (obtained by changing $x_{j}$ to 0 ) and one is in $S$ (obtained by changing $x_{n}$ to 0 ), thus $x \in A_{1}$. In the same way we prove that $\|x\|^{0}=1$ implies $x \in A_{1}$.

Now we proceed by induction noting that every $x \in\{0,1\}^{n}$ with $\|x\|^{1}=t+1 \geqslant 2$ has at least two neighbours $y$ with $\|y\|^{1}=t$ and analogously for $\|x\|^{0}=t+1 \geqslant 2$.

Lemma 8. Let $k, l \in \mathbb{N}_{0}, n=k+l+2$, and set $S=[*]^{k}[0]^{l+2}$ and $T=[0]^{k}[*]^{l} 11$ and $A_{0}=S \cup T$. Then

$$
A_{1} \supset[0]^{k+l} * *
$$

and

$$
\begin{aligned}
A_{t} \supset & \left\{x \in\{0,1\}^{n}: 1 \leqslant\|x\|^{01} \leqslant t-1\right\} \cup\left\{x \in\{0,1\}^{n}: 1 \leqslant\|x\|^{10} \leqslant t-1\right\} \cup \\
& \left\{x \in\{0,1\}^{n}: 1 \leqslant\|x\|^{00} \leqslant t-2\right\} \cup\left\{x \in\{0,1\}^{n}: 1 \leqslant\|x\|^{11} \leqslant t-2\right\}= \\
& \overline{\left([*]^{t-1}[0]^{k+l-t+1}\right)} 01 \cup \overline{\left([*]^{t-1}[0]^{k+l-t+1}\right)} 10 \cup \overline{\left([*]^{t-2}[0]^{k+l-t+2}\right)} 00 \cup \overline{\left([*]^{t-2}[0]^{k+l-t+2}\right)} 11
\end{aligned}
$$

for every $t \geqslant 2$.

Proof. An example of this case is shown in Figure 1. Clearly we have $\left\{[0]^{k+l+2},[0]^{k+l} 11\right\} \subset$ $S \cup T=A_{0}$. If $x \in\left\{[0]^{k+l} 01,[0]^{k+l} 10\right\}$ then $x$ has two neighbours in $A_{0}$ obtained by changing the value of one of the two last coefficients, thus $x \in A_{1}$.

We shall show that both $\|x\|^{01}=1$ and $\|x\|^{10}=1$ imply $x \in A_{2}$. Indeed, let $\|x\|^{01}=1$ and $x_{j}=1$ for some $1 \leqslant j \leqslant k+l$. Thus $x$ has two neighbours in $A_{1}$ : one is $[0]^{k+l} 01$ (obtained by changing $x_{j}$ to 0 ) and one is in $S \cup T$ (obtained by changing $x_{n}$ to 0 or $x_{n-1}$ to 1 , depending on whether $j \leqslant k$ or not), thus $x \in A_{1}$. In the same way we prove that $\|x\|^{10}=1$ implies $x \in A_{2}$.

Now we proceed by induction noting that every $x \in\{0,1\}^{n}$ with $\|x\|^{01}=t+1 \geqslant 2$ has at least two neighbours $y$ with $\|y\|^{01}=t$ and analogously for $\|x\|^{10}=t+1 \geqslant 2$.

Finally we show that, for every $t \geqslant 3$, both $\|x\|^{00}=t-2$ and $\|x\|^{11}=t-2$ imply $x \in A_{t}$. This is immediate as every such $x$ has two neighbours $y$ and $z$ with $\|y\|^{01}=t-2$ and $\|z\|^{10}=t-2$ which, by what we have just proved, belong to $A_{t-1}$.

In the next lemma we assume $k, l>0$ to avoid a trivial situation when $k=0$ or $l=0$ and $S \cup T=Q_{n}$.

Lemma 9. Let $k, l \in \mathbb{N}, n=k+l$, and set $S=[*]^{k}[0]^{l}$ and $T=[0]^{k}[*]^{l}$ and $A_{0}=S \cup T$. Then

$$
A_{t} \cap\left\{x \in\{0,1\}^{n}:\|x\|_{k, l} \geqslant t+2\right\}=\emptyset
$$

for every $0 \leqslant t \leqslant k+l-2$.

Proof. By induction on $t$, noting that $\|x\|_{k, l} \geqslant 2$ implies $x \notin S \cup T=A_{0}$, and that for every $x \in\{0,1\}^{n}$ with $\|x\|_{k, l}=t+1 \geqslant 3$, at most one of the neighbours $y$ of $x$ satisfies $\|y\|_{k, l}<t$ (there might be one neighbour $z$ with $\|z\|_{k, l}=0$ if $\sum_{j=1}^{k} x_{j}=1$ or $\left.\sum_{j=k+1}^{n} x_{j}=1\right)$. 


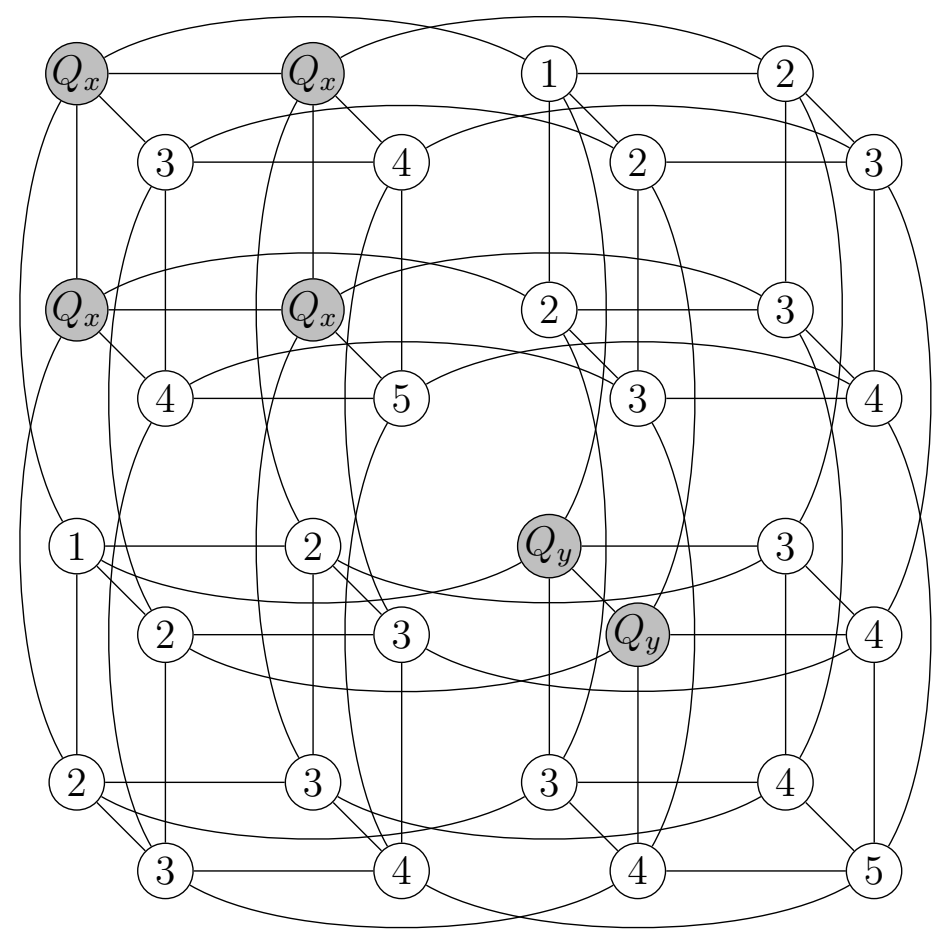

Figure 1: An example of spreading process on $Q_{5}$ for $x=* * 000$ and $y=00 * 11$. Here $n=m=5, k=2, l=1, p=0$ and $d=2$. Labels $1,2,3,4$ and 5 denote the time step at which vertices are infected. We see that here indeed $T_{Q_{n}}\left(Q^{x} \cup Q^{y}\right)=m-p=5$.

In the next lemma we avoid a trivial situation when $k=l=0$ and $S \cup T=Q_{1}$ by assuming without loss of generality that $k>0$.

Lemma 10. Let $k \in \mathbb{N}, l \in \mathbb{N}_{0}, n=k+l+1$, and set $S=[*]^{k}[0]^{l+1}$ and $T=[0]^{k}[*]^{l} 1$ and $A_{0}=S \cup T$. Then

$$
A_{t} \cap\left\{x \in\{0,1\}^{n}:\|x\|_{k}^{1} \geqslant t+1\right\}=\emptyset
$$

for every $0 \leqslant t \leqslant k+l-1$.

Proof. By induction on $t$, note first that $\|x\|_{k}^{1} \geqslant 1$ implies $x \notin S \cup T=A_{0}$. Now, for $t \geqslant 1$ assume that every $x$ with $\|x\|_{k}^{1} \geqslant t$ does not belong to $A_{t-1}$ and note that for every $x \in\{0,1\}^{n}$ with $\|x\|_{k}^{1} \geqslant t+1 \geqslant 2$, at most two of the neighbours $y$ of $x$ satisfy $\|y\|_{k}^{1}<t$. These two might be $w$ with $\|w\|_{k}^{0}=t+1$ obtained by changing $x_{n}$ to 0 and, if $\sum_{i=1}^{k} x_{i}=1$, $z$ with $\|z\|_{k}^{1}=0$. We claim that $z$, if it exists, is not in $A_{t-1}$.

Indeed, for $z$ to exist we must have $\sum_{i=1}^{k} x_{i}=1$ and $\sum_{i=k+1}^{k+l} x_{i}=t \geqslant 1$, so in particular $l \geqslant 1$. Let $z^{\prime}$ denote the vector obtained by swapping the first $k$ coordinates with the last $l$; that is,

$$
z^{\prime}=\left(z_{k+1}, \ldots, z_{k+l}, z_{1}, \ldots, z_{k}, z_{k+l+1}\right) .
$$

Then $\left\|z^{\prime}\right\|_{l}^{1} \geqslant t$, and so, by the case $t-1$ of the lemma (which we are assuming that we have already proved) applied to the sets $S^{\prime}=[*]^{l}[0]^{k+1}$ and $T=[0]^{l}[*]^{k} 1$, it follows that 
$z^{\prime}$ is not infected after $t-1$ steps of the bootstrap process with initial set $S^{\prime} \cup T^{\prime}$. By symmetry (since we have only reordered the coordinates), it follows that $z \notin A_{t-1}$, as required.

Thus $x$ can have at most one infected neighbour at time $t-1$ and therefore it does not belong to $A_{t}$.

In the next lemma we avoid a trivial situation when $k=l=0$ and $A_{1}=Q_{2}$ by assuming without loss of generality that $k>0$.

Lemma 11. Let $k \in \mathbb{N}, l \in \mathbb{N}_{0}, n=k+l+2$, and set $S=[*]^{k}[0]^{l+2}$ and $T=[0]^{k}[*]^{l} 11$ and $A_{0}=S \cup T$. Then

$$
A_{t} \cap\left\{x \in\{0,1\}^{n}:\|x\|_{k}^{01} \geqslant t \vee\|x\|_{k}^{10} \geqslant t\right\}=\emptyset
$$

for every $1 \leqslant t \leqslant k+l$, and

$$
A_{t} \cap\left\{x \in\{0,1\}^{n}:\|x\|_{k}^{11} \geqslant t-1\right\}=\emptyset
$$

for every $2 \leqslant t \leqslant k+l+1$.

Proof. Again, an example of this case is shown in Figure 1. Note first that $x \in[*]^{k+l} 01$, $x \in[*]^{k+l} 10$ and $\|x\|_{k}^{11} \geqslant 1$ each imply $x \notin S \cup T=A_{0}$. Also both $\|x\|_{k}^{01} \geqslant 1$ and $\|x\|_{k}^{10} \geqslant 1$ imply $x \notin A_{1}$ as such $x$ can have at most one neighbour $y$, obtained by changing respectively $x_{n}$ and $x_{n-1}$ to 0 , in $A_{0}$. Similarily $\|x\|_{k}^{11} \geqslant 1$ implies $x \notin A_{1}$ as such $x$ can have at most one initially infected neighbour $y \in[*]^{k+l} 11$ with $\|y\|_{k}^{11}=0$, while all of its other neighbours $y$ have either $\|y\|_{k}^{01} \geqslant 1,\|y\|_{k}^{10} \geqslant 1$ or $\|y\|_{k}^{11} \geqslant 1$. What is more, $\|x\|_{k}^{11} \geqslant 1$ implies $x \notin A_{2}$ as all these neighbours are not even in $A_{1}$.

Now, for $t \geqslant 1$ assume that every $x$ with $\|x\|_{k}^{01} \geqslant t$ or $\|x\|_{k}^{10} \geqslant t$ does not belong to $A_{t}$ and that every $x$ with $\|x\|_{k}^{11} \geqslant t$ does not belong to $A_{t+1}$.

Note that for every $x \in\{0,1\}^{n}$ with $\|x\|_{k}^{01} \geqslant t+1$, at most three of its neighbours $y$ satisfy $\|y\|_{k}^{01}<t$ which is a necessary condition to belong to $A_{t}$. One of these three neighbours is $v$ with $\|v\|_{k}^{11}=t+1 \geqslant 2$ obtained by changing $x_{n-1}$ to 1 , thus also $v \notin A_{t}$. The other two might be $w$ with $\|w\|_{k}^{00}=t+1 \geqslant 2$ obtained by changing $x_{n}$ to 0 and, if $\sum_{i=1}^{k} x_{i}=1, z$ with $\|z\|_{k}^{01}=0$. We claim that $z$, if it exists, is not in $A_{t}$.

Indeed, for $z$ to exist we must have $\sum_{i=1}^{k} x_{i}=1$ and $\sum_{i=k+1}^{k+l} x_{i}=t \geqslant 1$, so in particular $l \geqslant 1$. We now follow steps similar to those in the proof of Lemma 10: let $z^{\prime}$ denote the vector obtained by swapping the first $k$ coordinates with the last $l$; that is,

$$
z^{\prime}=\left(z_{k+1}, \ldots, z_{k+l}, z_{1}, \ldots, z_{k}, z_{k+l+1}, z_{k+l+2}\right) .
$$

Then $\left\|z^{\prime}\right\|_{l}^{01} \geqslant t$, and so, by the case $t$ of the lemma (which we are assuming that we have already proved) applied to the sets $S^{\prime}=[*]^{l}[0]^{k+2}$ and $T=[0]^{l}[*]^{k} 11$, it follows that $z^{\prime}$ is not infected after $t$ steps of the bootstrap process with initial set $S^{\prime} \cup T^{\prime}$. By symmetry (since we have only reordered the coordinates), it follows that $z \notin A_{t}$, as required. Thus $x$ can have at most one infected neighbour at time $t$ and therefore it does not belong to $A_{t+1}$. 
Finally, every $x \in\{0,1\}^{n}$ with $\|x\|_{k}^{11}=t+1 \geqslant 2$ has at most one neighbour $y$ with $\|y\|_{k}^{11}=0$ which might be in $A_{t+1}$. All other neighbours of $x$ are either $v$ with $\|v\|_{k}^{11}=t, w$ with $\|w\|_{k}^{01}=t+1$ or $y$ with $\|z\|_{k}^{10}=t+1$, neither of which is in $A_{t+1}$. Thus $x \notin A_{t+2}$.

Let us now summarize what we know about the spreading time of $Q_{k}^{x} \cup Q_{l}^{y}$ in $Q_{n}$ for particular choices of $x$ and $y$.

Corollary 12. For vectors $x, y \in\{0,1, *\}^{n}$ such that $\operatorname{dim}(x)=k, \operatorname{dim}(y)=l, \operatorname{dim}(x \vee$ $y)=m$, where $k, l<m \leqslant n, d(x, y)=d \leqslant 2$, and such that $\left|\left\{i: x_{i}=y_{i}=*\right\}\right|=p$, the spreading time of $Q^{x} \cup Q^{y}$ in $Q_{n}$ is given by

$$
T_{Q_{n}}\left(Q^{x} \cup Q^{y}\right)= \begin{cases}m-p, & \text { if } d=2 \text { and }(k, l) \neq(m-2, m-2), \\ m-p-1, & \text { otherwise. }\end{cases}
$$

Proof. By the symmetry of the hypercube, without loss of generality assume that $l \leqslant k$ and that

$$
x=[*]^{k}[0]^{n-k}, y=[*]^{p}[0]^{k-p}[*]^{l-p}[1]^{d}[0]^{n-m} .
$$

Note that we have $m=p+(k-p)+(l-p)+d=k+l-p+d$. Note also that both the first $p$ coordinates, for which $x_{i}=y_{i}=*$, and the last $n-m$ coordinates, for which $x_{i}=y_{i}=0$, do not matter when we look at the spreading times since infection process will behave like $2^{p}$ parallel infection processes on identical $(m-p)$-dimensional subcubes which do not influence each other.

$d=0$ : by Lemma 6 we have $T_{Q_{n}}\left(Q^{x} \cup Q^{y}\right) \leqslant k-p+l-p-1=m-p-1$. Also, noting that the maximal value of the norm $\|\cdot\|_{k, l}$ in Lemma 9 is $k+l, T_{Q_{n}}\left(Q^{x} \cup Q^{y}\right) \geqslant$ $k-p+l-p-1=m-p-1$.

$d=1$ : by Lemma 7 we have $T_{Q_{n}}\left(Q^{x} \cup Q^{y}\right) \leqslant k-p+l-p=m-p-1$. Also, noting that the maximal value of the norm $\|\cdot\|_{k}^{1}$ in Lemma 10 is $k+l, T_{Q_{n}}\left(Q^{x} \cup Q^{y}\right) \geqslant$ $k-p+l-p=m-p-1$. Note that if $k-p=l-p=0$ then $m-p=1$ and the formula on $T_{Q_{n}}\left(Q^{x} \cup Q^{y}\right)$ is also correct.

$d=2$ : if $(k, l)=(m-2, m-2)$ then $p=m-2$, infection takes exactly one step and the formula on $T_{Q_{n}}\left(Q^{x} \cup Q^{y}\right)$ is correct. In the other case, by Lemma 8, $T_{Q_{n}}\left(Q^{x} \cup Q^{y}\right) \leqslant$ $k-p+l-p+2=m-p$. Also, noting that the maximal value of the norm $\|\cdot\|_{k}^{11}$ in Lemma 11 is $k+l, T_{Q_{n}}\left(Q^{x} \cup Q^{y}\right) \geqslant k-p+l-p+2=m-p$ (see Figure 1 ).

The next lemma will be used later to simplify a recurrence formula we shall obtain for $M(n)$.

Lemma 13. Let $a(1)=0, a(2)=1, a(3)=3$ and for $n \geqslant 4$

$$
a(n)=\max \left\{\begin{array}{l}
a(n-2)+n, \\
a(n-3)+2 n-3 .
\end{array}\right.
$$

Then $a(n)=a(n-3)+2 n-3$ for all $n \geqslant 4$. 
Proof. First, we immediately see that $a(4)=a(1)+5=a(2)+4=5$. Similarly, it can be trivially checked that the lemma holds for $4 \leqslant n \leqslant 6$.

Now, we prove the lemma by induction. For $n \geqslant 4$ we assume that it holds for $n, n+1$ and $n+2$, and for $n+3$ we obtain

$$
\begin{aligned}
& a(n+3)=\max \{a(n)+2(n+3)-3, a(n+1)+n+3\}= \\
& \quad \max \{a(n-3)+4 n, a(n-2)+3 n+2\}=a(n-3)+4 n=a(n)+2(n+3)-3,
\end{aligned}
$$

where the third equality follows from the fact that

$a(n-3)+4 n=a(n-3)+2 n-3+(2 n+3) \geqslant a(n-2)+n+(2 n+3)>a(n-2)+3 n+2$.

Let us prove a recursion formula for the maximal percolation time which we shall later use to give a closed-form expression for $M(n)$.

Theorem 14. We have $M(1)=0, M(2)=1, M(3)=3, M(4)=5$ and for $n \geqslant 5$

$$
M(n)=\max \left\{\begin{array}{l}
M(n-2)+n, \\
M(n-3)+2 n-3 .
\end{array}\right.
$$

Proof. The values of $M(n)$ for $n \leqslant 4$ can be found by exhaustive search. The maximal percolation time can be obtained with the following sets of sites:

$$
n=1:\{0,1\}, n=2:\{00,11\}, n=3:\{000,110,001\}, n=4:\{0000,1100,0111\} .
$$

We shall first prove that for $n \geqslant 5$ the following holds.

$$
M(n) \geqslant \max \left\{\begin{array}{l}
M(n-2)+n, \\
M(n-3)+2 n-3 .
\end{array}\right.
$$

Consider the following two ways of infecting $Q_{n}$. Note that the second way corresponds to the optimal family briefly described at the end of Section 1.

1. Let $A^{n-2}$ be a set that internally spans the hypercube $Q_{n-2}^{x}$ for $x=[*]^{n-2} 00$ in time $M(n-2)$ and such that the site $[0]^{n}$ becomes infected at time $M(n-2)$. Let $\tilde{A}^{n-2}=A^{n-2} \cup[0]^{n-2} 11$; then $\left\langle\tilde{A}^{n-2}\right\rangle=Q_{n}$ and, by Corollary 12 case $d=2$, $T_{Q_{n}}\left(\tilde{A}^{n-2}\right)=M(n-2)+n$,

2. Let $A^{n-3}$ be a set that internally spans the hypercube $Q_{n-3}^{x}$ for $x=[*]^{n-3} 000$ in time $M(n-3)$ and such that the site $[0]^{n}$ becomes infected at time $M(n-3)$. Let $\tilde{A}^{n-3}=A^{n-3} \cup[0]^{n-3} 110 \cup[1]^{n}$ (note that we require $n \geqslant 5$ here so that the distance between $[0]^{n-3} 110$ and $[1]^{n}$ is $\left.\geqslant 3\right)$. Then clearly $\left\langle\tilde{A}^{n-3}\right\rangle=Q_{n}$. The set of sites infected after $M(n-3)$ steps is $[*]^{n-3} 000 \cup[0]^{n-3} 110 \cup[1]^{n}$. By Lemmas 8 and 11 
after $M(n-3)+n-2$ steps all neighbours $y$ of $[1]^{n-1} 0$ having norm $\|y\|^{110}=n-4$, $\|y\|^{010}=n-3$ or $\|y\|^{100}=n-3$ (i.e., all sites at distance two from $[1]^{n}$ in $[*]^{n-1} 0$ ), become infected at time exactly $M(n-3)+n-2$, so at time $M(n-3)+n-2$ the only infected site in $[*]^{n-1} 1$ is $[1]^{n}$. Thus by Corollary 12 it takes at least $n-1$ more steps to fully infect $Q_{n}$, and so $T_{Q_{n}}\left(\tilde{A}^{n-3}\right)=M(n-3)+2 n-3$.

Now let us prove an upper bound on $M(n)$, i.e.,

$$
M(n) \leqslant \max \left\{\begin{array}{l}
M(n-2)+n, \\
M(n-3)+2 n-3 .
\end{array}\right.
$$

Let $A$ be a set spanning the hypercube $Q_{n}$ for $n \geqslant 5$. Let

$$
Q_{0}=Q_{i_{1}}^{x_{i_{1}}} \subset Q_{i_{2}}^{x_{i_{2}}} \subset \ldots \subset Q_{i_{t-1}}^{x_{i_{t-1}}} \subset Q_{i_{t}}^{x_{i_{t}}}=Q_{n}
$$

be a building sequence of the hypercube. Let $Q_{m_{1}}^{z_{m_{1}}}, Q_{m_{2}}^{z_{m_{2}}}, \ldots, Q_{m_{t-2}}^{z_{m_{t-2}}}, Q_{m_{t-1}}^{z_{m_{t-1}}}$ be the cubes that merge with cubes $Q_{i_{j}}^{x_{i_{j}}}$ like in the statement of Lemma 4. Recall that for each $1 \leqslant j \leqslant t-1$ we have $i_{j} \geqslant m_{j}$. As adding sites to a set that spans $Q_{n}$ cannot increase its spreading time we may assume that $A$ is a minimal under containment set spanning $Q_{n}$. Therefore $i_{t-1}<n$. Let us consider the possible scenarios of the infection process started from $A$.

1. If $i_{t-1} \leqslant n-2$ then, by Lemma 5 , after at most $M\left(i_{t-1}\right) \leqslant M(n-2)$ time steps both $Q_{i_{t-1}}^{x_{i_{t-1}}}$ and $Q_{m_{t-1}}^{z_{m_{t-1}}}$ are fully infected. Then, since $\left\langle Q_{i_{t-1}}^{x_{i_{t-1}}} \cup Q_{m_{t-1}}^{z_{m_{t-1}}}\right\rangle=Q_{n}$, by Corollary 12 after at most $n$ more steps we have percolation. Thus in this case

$$
T_{Q_{n}}(A) \leqslant M(n-2)+n .
$$

2. If $i_{t-1}=n-1$ and $i_{t-2}=n-2$ then (since $Q_{i_{t-1}}^{x_{i_{t-1}}}$ is internally spanned) we must have some site $v \in A \cap Q_{m_{t-2}}^{z_{m_{t-2}}}$ such that $d\left(x_{i_{t-2}}, v\right)=1$ and $\left\langle Q_{i_{t-2}}^{x_{i_{t-2}}} \cup v\right\rangle=Q_{i_{t-1}}^{x_{i_{t-1}}}$. Also, there must exist some site $w \in A \cap Q_{m_{t-1}}^{z_{m_{t-1}}}$ such that $\left\langle Q_{i_{t-1}}^{x_{i_{t-1}}} \cup w\right\rangle=Q_{n}$. Note that, since $i_{t-2}=n-2$, either $d\left(x_{i_{t-2}}, w\right)=1$ or $d\left(x_{i_{t-2}}, w\right)=2$. Let us consider these situations separately.

If $d\left(x_{i_{t-2}}, w\right)=2$ then $\left\langle Q_{i_{t-2}}^{x_{i_{t-2}}} \cup w\right\rangle=Q_{n}$ which contradicts the minimality of $A$, as $\langle A \backslash\{v\}\rangle=Q_{n}$.

If $d\left(x_{i_{t-2}}, w\right)=1$ then, without loss of generality, we have

$$
x_{i_{t-2}}=[*]^{n-2} 00, x_{i_{t-2}} \vee v=[*]^{n-1} 0, x_{i_{t-2}} \vee w=[*]^{n-2} 0 * .
$$

Clearly, after at most $M(n-2)$ time steps the cube $Q_{i_{t-2}}^{x_{i_{t-2}}}$ is fully infected. Then, by Corollary 12 case $d=1$, after at most $(n-1)-1=n-2$ more steps both $Q_{n-1}^{x_{i_{t-2}} \vee v}$ and $Q_{n-1}^{x_{i_{t-2}} \vee w}$ are fully infected. Clearly (or, by Corollary 12 case $d=0$ ), after one more step we have percolation. Thus in this case

$$
T_{Q_{n}}(A) \leqslant M(n-2)+(n-2)+1=M(n-2)+n-1 .
$$


3. If $i_{t-1}=n-1, i_{t-2} \leqslant n-3$ and $d\left(x_{i_{t-2}}, z_{m_{t-2}}\right) \leqslant 1$ then after at most $M(n-3)$ time steps both $Q_{i_{t-2}}^{x_{i_{t-2}}}$ and $Q_{m_{t-2}}^{z_{m_{t-2}}}$ are fully infected. Then, by Corollary 12 case $d=0$ or $d=1$, after at most $i_{t-1}-1=n-2$ more time steps $Q_{i_{t-1}}^{x_{i_{t-1}}}$ is fully infected. Since $i_{t-1}=n-1$ we must have $d\left(x_{i_{t-1}}, z_{m_{t-1}}\right) \leqslant 1$ so, again by Corollary 12 case $d=0$ or $d=1$, after at most $n-1$ more time steps we have percolation. Thus in this case

$$
T_{Q_{n}}(A) \leqslant M(n-3)+n-2+n-1=M(n-3)+2 n-3 .
$$

4. If $i_{t-1}=n-1, i_{t-2}=n-3, d\left(x_{i_{t-2}}, z_{m_{t-2}}\right)=2$ and $m_{t-2}=n-3$ then after at most $M(n-3)$ time steps both $Q_{i_{t-2}}^{x_{i t-2}}$ and $Q_{m_{t-2}}^{z_{m_{t-2}}}$ are fully infected. Then, by Corollary 12 case $d=2,(k, l)=(m-2, m-2)$, after at most $i_{t-1}-1=n-2$ more time steps $Q_{i_{t-1}}^{x_{i_{t-1}}}$ is fully infected. Again, since $i_{t-1}=n-1$ we must have $d\left(x_{i_{t-1}}, z_{m_{t-1}}\right) \leqslant 1$ so, again by Corollary 12 case $d=0$ or $d=1$, after at most $n-1$ more time steps we have percolation. Thus in this case again

$$
T_{Q_{n}}(A) \leqslant M(n-3)+n-2+n-1=M(n-3)+2 n-3 .
$$

5. Finally we consider the case $i_{t-1}=n-1, i_{t-2} \leqslant n-3, d\left(x_{i_{t-2}}, z_{m_{t-2}}\right)=2$ and $m_{t-2}<n-3$. Without loss of generality

$$
x_{i_{t-1}}=[*]^{n-1} 0, x_{i_{t-2}}=[*]^{i_{t-2}}[0]^{n-i_{t-2}}, z_{m_{t-2}}=[*]^{p}[0]^{i_{t-2}-p}[*]^{m_{t-2}-p} 110,
$$

with $i_{t-2}-p>0$, which follows from $m_{t-2}<n-3$. Again after at most $M(n-3)$ time steps both $Q_{i_{t-2}}^{x_{i_{t-2}}}$ and $Q_{m_{t-2}}^{z_{m_{t-2}}}$ are fully infected so let us assume it is the case and see how the process goes from this point.

Even if we limit our attention only to the initially infected sites in $Q_{i_{t-1}}^{x_{t_{t-1}}}$ then, by Lemma 8 , at most two sites in $Q_{i_{t-1}}^{x_{i_{t-1}}}, s=[1]^{n-1} 0$ with $\|s\|^{110}=n-3$ and $t=[1]^{n-3} 000$ with $\|t\|^{000}=n-3$, are not yet infected after $(n-1)-1=n-2$ additional steps. Let $\tilde{y} \in A$ be such that $\tilde{y} \in[*]^{n-1} 1$. Such $\tilde{y}$ must exist as otherwise no site in $[*]^{n-1} 1$ would ever become infected. If $d(\tilde{y}, s), d(\tilde{y}, t) \neq 2$ then all neighbours of $\tilde{y}$ in $[*]^{n-1} 1$ have their neighbour in $[*]^{n-1} 0$ already infected at time $M(n-3)+n-2$ thus at latest at this moment the infection of the subcube $[*]^{n-1} 1$ starts with $\tilde{y}$ as its "seed" and by Corollary 12 case $d=1$ takes at most $n-1$ steps so again

$$
T_{Q_{n}}(A) \leqslant M(n-3)+n-2+n-1 \leqslant M(n-3)+2 n-3 .
$$

If $d(\tilde{y}, s)=2$ or $d(\tilde{y}, t)=2$ (which strongly constraints our possible choices of $\tilde{y}$ ) then let $y^{*} \in[*]^{n-1} 1$ be such that $d\left(y^{*}, \tilde{y}\right)=1, d\left(y^{*}, s\right)=d\left(y^{*}, t\right)=3$ (we do not require $y^{*} \in A$ ). Note that such site can always be found as $d(s, t)=2$. Note also that we must have $\left\|y^{*}\right\|^{011}=n-4$ or $\left\|y^{*}\right\|^{101}=n-4$. Let $w^{*}$ be the neighbour of $y^{*}$ in $[*]^{n-1} 0$. Clearly $d\left(w^{*}, s\right)=d\left(w^{*}, t\right)=2$ since $w^{*}$ is obtained from $y^{*}$ by changing $y_{n}^{*}$ to 0 . Also, $\left\|w^{*}\right\|^{010}=n-4$ or $\left\|w^{*}\right\|^{100}=n-4$. 
Again by Lemma 8, in the process started from $Q_{i_{t-2}}^{x_{i_{t-2}}} \cup Q_{m_{t-2}}^{z_{m_{t-2}}}$ and constrained to $Q_{i_{t-1}}^{x_{i_{t-1}}}, w^{*}$ becomes infected after at most $(n-1)-2=n-3$ steps. From this follows that $y^{*}$ becomes infected after at most $n-2$ steps (recall that it is a neighbour of $w^{*}$ and of an initially infected site $\tilde{y}$ ). Also by Lemma 8 , all sites $v^{*}$ in $[*]^{n-1} 0$ at distance 2 from $y^{*}$, which must have either $0<\left\|v^{*}\right\|^{010} \leqslant n-3$, $0<\left\|v^{*}\right\|^{100} \leqslant n-3,\left\|v^{*}\right\|^{000}=n-4$ or $\left\|v^{*}\right\|^{110}=n-4$, are as well infected after at most $n-2$ steps. Therefore the infection of the subcube $[*]^{n-1} 1$ starts in the worst case after $M(n-3)+n-2$ steps with $y^{*}$ as its "seed" and it spreads undisturbed by the states of $s$ and $t$. Thus by Corollary 12 case $d=1$ it can take at most $n-1$ additional time steps. Thus once again

$$
T_{Q_{n}}(A) \leqslant M(n-3)+n-2+n-1 \leqslant M(n-3)+2 n-3 .
$$

This completes the proof.

From Lemma 13 and Theorem 14 we get the following corollary.

Corollary 15. We have $M(1)=0, M(2)=1, M(3)=3$ and for $n \geqslant 4, M(n)=$ $M(n-3)+2 n-3$.

We are now ready to prove our main result.

Proof of Theorem 1. Theorem holds for $n \in\{1,2,3\}$. Assume that it holds for $n-3$. By Corollary 15 we obtain

$$
M(n)=M(n-3)+2 n-3=\left\lfloor\frac{(n-3)^{2}}{3}\right\rfloor+2 n-3=\left\lfloor\frac{n^{2}}{3}-2 n+3\right\rfloor+2 n-3=\left\lfloor\frac{n^{2}}{3}\right\rfloor .
$$

\section{Further questions}

In this paper we find the maximal percolation time in the $n$-dimensional hypercube under 2-bootstrap percolation. It is natural to consider a similar problem on other families of graphs.

Problem 16. Determine the precise value or the order of magnitude of the maximal percolation time in $[n]^{d}$ for $n$ and $d$ constant or tending to infinity.

Another very interesting question asks how many small percolating subsets can be found in a hypercube. A simple result from Balogh and Bollobás [2] says that in 2bootstrap percolation every percolating set in $Q_{n}$ must contain at least $\left\lceil\frac{n}{2}\right\rceil+1$ sites so the question can be formulated as follows.

Problem 17. For $\epsilon>0$ what is the number of percolating sets of size $\left(\frac{1}{2}+\epsilon\right) n$ in $Q_{n}$ ?

This problem can be also interpreted as determining the probability that a random $\left(\frac{1}{2}+\epsilon\right) n$-set of vertices of $Q_{n}$ percolates. Note that in our proof of the value of $M(n)$ we show that the maximal spreading time is obtained for a set of size roughly $\frac{2 n}{3}$. 


\section{Acknowledgements}

I would like to thank Béla Bollobás for bringing the problem to my attention. I am also very grateful to the anonymous referee for all the comments and corrections that helped me significantly improve the quality of this paper.

\section{References}

[1] M. Aizenman and J. Lebowitz. Metastability effects in bootstrap percolation. Journal of Physics A, 21:3801-3813, 1988.

[2] J. Balogh and B. Bollobás. Bootstrap percolation on the hypercube. Probability Theory and Related Fields, 134:624-648, 2006.

[3] J. Balogh, B. Bollobás and R. Morris. Bootstrap percolation in three dimensions. Annals of Probability, 37:1329-1380, 2009.

[4] J. Balogh, B. Bollobás and R. Morris Majority bootstrap percolation on the hypercube. Combinatorics, Probability and Computing, 18:17-51, 2009.

[5] J. Balogh, B. Bollobás and R. Morris Bootstrap percolation in high dimensions. Combinatorics, Probability and Computing, 37:643-692, 2010.

[6] J. Balogh and G. Pete. Random disease on the square grid. Random Structures and Algorithms, 13:409-422, 1998.

[7] F. S. Benevides and M. Przykucki. Maximum percolation time in two-dimensional bootstrap percolation. Preprint, 2011.

[8] J. Chalupa, P. L. Leath and G. R. Reich. Bootstrap percolation on a Bethe latice. Journal of Physics C, 12:L31-L35, 1979.

[9] A. E. Holroyd. Sharp metastability threshold for two-dimensional bootstrap percolation. Probability Theory and Related Fields, 125:195-224, 2003.

[10] R. Morris. Minimal percolating sets in bootstrap percolation. The Electronic Journal of Combinatorics, 16(1)\#R2:1-20, 2009.

[11] J. von Neumann. Theory of Self-Reproducing Automata. University of Illinois Press, 1966.

[12] E. Riedl. Largest minimal percolating sets in hypercubes under 2-bootstrap percolation. The Electronic Journal of Combinatorics, 17(1)\#R80:1-13, 2010.

[13] S. Ulam. Random processes and transformations. In Proceedings of the International Congress of Mathematicians, Vol. 2, Cambridge, Massachusetts, August 30September 6, 1950, 264-275, 1952. 